TITLE: Enablers and barriers to seeking help for a postpartum mood disorder RUNNING HEAD: "Enablers and Barriers"

Michelle Foulkes, RN BA BScN MSc CPMHN(c) PhD(c) NP-PHC(student)

Advanced Practice Nurse

Inpatient Psychiatry and Eating Disorders Program

401 Smyth Road

Ottawa, ON K1H 8L1 


\title{
Enablers and barriers to seeking help for a postpartum mood disorder
}

Key words: postpartum mood disorders, help-seeking behaviours, barriers, enablers

\begin{abstract}
Objective: To explore the barriers and enablers identified by women experiencing a postpartum mood disorder (PPMD) that both preclude and facilitate their help-seeking behaviours for this often devastating illness.

Design: A qualitative study using a grounded theory approach

Setting: Well-Baby Clinics offered through the Public Health Department, Early Years Centres, Mothercraft and a Parent Resource Centre in a large Canadian city.

Participants: Ten women who had either been formally diagnosed as having a PPMD or who self-identified as experiencing a constellation of symptoms indicative of a PPMD Methods: Interviews that were transcribed verbatim and analyzed using a grounded theory approach as described by Strauss and Corbin (1998).

Results: The core category of 'Having postpartum' captured the essence of women's experiences in seeking help for a PPMD. Women identified four main stressors that contributed to their development of a PPMD, two barrier categories and an enabler category which influenced their help-seeking behaviours. Through navigation of both formal and informal help, women were able to begin the journey to reclaim the mothering soul they had lost to mental illness.
\end{abstract}

Conclusions: Pregnancy, birth and becoming a mother collectively represent a critical period of physical and emotional upheaval in a woman's life. The need for a holistic care approach that supports the emotional and physical health of the dyad is imperative. 


\section{Callouts}

1. Postpartum mood disorders represent a complex class of illnesses that may result in serious implications for new mothers but can also consume those around her.

2. This use of the label of 'having postpartum' provides safety for women in a society where the stigma around mental illness remains deeply entrenched.

3. Women need their physical health monitored appropriately but also need emotionally supportive care that values and honours the needs of both women and their babies. 
The development of maternal mood disturbances in the postpartum period is not a

2 newly emergent phenomenon but rather has been noted as early as the $5^{\text {th }}$ century B.C. in

3 the works of Hippocrates (Ugarriza, 2002). In current times, several distinct mood and

4 anxiety disorders have been identified during the postnatal period each with unique

5 presentations and symptoms including maternal "blues", postpartum depression (PPD),

6 postpartum psychosis, postpartum anxiety disorders, bipolar disorders and post traumatic

$7 \quad$ stress disorder secondary to birth trauma (Beck \& Driscoll, 2006).

8 Although women may experience a broad range of psychiatric symptoms following

9 birth and while mothering, maternal "blues", PPD and postpartum psychosis collectively

10 fall under the umbrella term of postpartum mood disorders (PPMDs) (Beck \& Driscoll,

11 2006) although it should be understood that they are distinct disorders requiring different

12 intervention and support. The focus of this study has been on women's experiences with

13 seeking help for a mood disturbance (PPMD) that may have developed after giving birth.

14 Where appropriate, the term PPD has been substituted for PPMD to accurately reflect the

15 terminology used in the literature reviewed. Post traumatic stress disorder secondary to

16 birth trauma will also be considered given its relevance to the findings of this study

17 The incidence rate for PPD varies anywhere between 10-40\% worldwide

18 (Holopainen, 2002). More commonly however, it is estimated that approximately 10-

$1915 \%$ of new mothers experience a mood disorder and is the most frequently occurring

20 illness experienced by women in the puerperium (Barr, J.A., 2006; Pearson, R.M.,

21 Cooper, R.M., Penton-Voak, I.S., Lightman, S.L. \& Evans, J., 2010). Diagnosis for a

22 PPD is currently subsumed under the Diagnostic and Statistical Manual of Mental

23 Disorders, $4^{\text {th }}$ Edition (DSM-IV-TR) criteria for major depression with a modifier that 
24 specifies symptoms must begin within four weeks of delivery (American Psychiatric

25 Association, 2000). In practice, however, it is generally accepted that PPD may develop

26 any time in the first year after giving birth (Goodman, 2004).

27 Women may also experience posttraumatic stress disorder secondary to birth

28 trauma which presents with a unique configuration of symptoms that may include

29 extreme fear, panic, dissociation, and flashbacks (Beck \& Driscoll, 2006). Within the

30 literature, it is estimated that between 1.5 and $6 \%$ of mother's experience a posttraumatic

31 stress disorder (PTSD) after childbirth (Beck, 2004). PTSD after childbirth does not have

32 a distinct diagnostic category in the DSM-IV-TR but the experience can be defined as a

33 traumatic event resulting in PTSD which may manifest itself as profound anxiety,

34 depression, hopelessness, fear, and constant arousal deeply affecting a woman's mental

35 health. Because of the affective elements of the disorder, it does share some

36 commonalities with PPMDs.

37 For women experiencing a PPMD, symptoms may persist for months and even

38 years if left untreated with an increased incidence of self-medication for relief of the

39 debilitating symptoms that define these disorders (e.g. alcohol abuse) (Beck \& Driscoll,

40 2006). This single psychiatric event may also be the launch of continuing and recurrent

41 mental illness over the long-term and significantly reduce a woman's functioning

42 capacity and her overall quality of life (Forman, Videbech, Hedegaard, Salvig \& Secher,

43 2000). Women who experience a PPMD also have a 50\% higher risk of developing it

44 again in subsequent pregnancies (Gold, 2002; The Maternity Center Association, 2002).

45 For her infant, both short-term and long-term sequelae from exposure to maternal

46 mood disturbances may ensue with an increased risk for neglect, higher accident and 
47 hospitalization rates, increased incidence of non-organic failure to thrive, social and

48 affective disorders and cognitive delays noted (Field, T., 2010, Gao, W., Paterson, J.,

49 Abbott, M., Carter, S., Iusitini, L., 2007; Tough, S.C., Siever, J.E., Leew, S., Johnston,

50 D.W., Benzies, K., \& Clark, D., 2008). Mothers with mood disturbances often present

51 with a flat affect and show less contingency responsiveness towards their infants (Beck \&

52 Driscoll, J.W., 2006). Overtime, infants of these mentally ill mothers develop an

53 interaction style that "mimics" that of their mothers (Cohen, L.S. \& Nonacs, R.M., 2005).

54 By 12-18 months of age, cognitive delays, particularly for male children, are often

55 apparent (Tronick, E., 2007).

56 Longitudinal studies have evaluated the emotional, cognitive and behavioural

57 effects of exposure to maternal mood disturbances and have indicated that the effects are

58 enduring. By age 11, both male and female children present with lower IQ scores,

59 attention problems, conduct disorders, and difficulties in mathematical reasoning (Hay,

60 Pawlby, Sharp Asten, Mills \& Kumer, 2001). For the family as a whole, a PPMD can

61 exert significant influence on the dynamics within the unit and ultimately may result in

62 increased marital discord further disabling the functioning, development, and quality of

63 life for all family members (Beck, 1999; Meighan, Davis, Thomas \& Droppleman, 1999).

64 Therefore, PPMD's represent a complex and multi-faceted class of illnesses that may

65 result in serious implications for new mothers but can also cascade to consume and

66 threaten those around her (Gold, 2002).

67 Because the presence of a PPMD can have such a significantly negative impact on

68 health, a qualitative study was proposed that asked the central question: "What are the

69 perceived barriers and enablers identified by women that preclude and facilitate their 
70 seeking help for a postpartum mood disorder?" This broad and open-ended research

71 question directed the research process and served as the focus around which all data were

72 collected and analyzed to ultimately allow us a better understanding of the enablers and

73 barriers for women to seeking help for a PPMD given the deleterious consequences on

74 women, children and families.

\section{Method}

At present, our understanding of help-seeking behaviours in women experiencing a

77 PPMD remains largely undeveloped. Grounded theory is a recommended method of

78 inquiry for areas where little previous research has been done on the phenomena of

79 interest (Strauss \& Corbin, 1998). The use of a grounded theory approach is also

80 compatible with nursing's epistemological goals of generating nursing knowledge,

81 promoting theory development, and informing practice (McCreaddie \& Payne 2010;

82 Meleis, 2005). Therefore, the use of grounded theory as a means of increasing our

83 knowledge of the perceived barriers and enablers that direct women's help-seeking

84 behaviours is congruent with the purpose and objectives of this study.

85 Data Collection: Following ethics approval, posters and flyers were distributed to

86 several Well-Baby Clinics offered through the Public Health Department (PHD), two

87 Ontario Early Year's Centres, Mothercraft, and a Parent Resource Centre where a

88 Postpartum Depresion Support Group (M.O.M.'s) was based. A public health nurse

89 (PHN) served as a liaison to direct and facilitate the recruitment of interested participants

90 at Well-Baby Clinics. The inclusion criteria for participation in the study included: a)

91 women had to be at least 18 years of age b) English speaking c) had given birth to a

92 healthy, full-term infant within the last 24 months d) no history of a serious pre-existing 
93 psychiatric illness prior to the development of a PPMD e) been formally diagnosed as

94 having a PPMD by a physician or f) not have been formally diagnosed with a PPMD by a

95 physician but who were experiencing symptoms which they identified as intrusive and

96 disabling to their functioning as mothers. All the women who self-identified as having a

97 PPMD did experience some combination of symptoms which included extreme sadness

98 and tearfulness, irritability, thoughts of harming themselves or their infants, difficulty

99 concentrating, weight loss or gain that was not explained by normal somatic changes

100 associated with the postpartum period, or sleep difficulties which were not related to

101 infant care issues.

102 Recruitment to the study began in October 2003 and continued through to

103 February 2004 when it was determined that theoretical saturation had been achieved. In

104 the end, 10 women who met the inclusion criteria participated in the study. A purposeful

105 sampling technique was used at the beginning of the research project which was then

106 superseded by theoretical sampling (Bryant \& Charmaz, 2007). Two participants were

107 recruited through the PHD, 2 through the postpartum depression support group

108 (M.O.M.'s), 1 through referral by a doula who had seen the recruitment poster and 5

109 through snowballing.

110 A semi-structured interview guide was developed to provide some direction to the

111 researcher but during interviews women were invited to openly tell their stories to ensure

112 their voices were heard and their experiences shared without a rigid adherence to the

113 interview guide (Charmaz, 2006). Each interview started with the broad opening

114 question of "tell me what you pregnancy was like" which served as a port of entry into

115 this significant experience in a woman's life. All interviews were completed by a single 
116 researcher and conducted in the participant's homes at their request. At the end of the

117 first interview, all of the women were asked and agreed to participate in a second

118 interview to provide a 'member check' and validate the findings following analysis of the

119 data. All taped interviews were transcribed verbatim by the researcher to ensure further

120 immersion in the data. Observational notes and journal reflections were maintained

121 throughout data collection and analysis to supplement the in-depth interviews (Charmaz,

122 2006). These served as important resources during data analysis to stimulate further

123 ideas and discussion between the researcher and her supervisor as well as the thesis

124 committee.

125 Data Analysis: Data analysis occurred through the 'constant comparative method'

126 with data being analyzed in a circular fashion in keeping with the methods described by

127 Strauss and Corbin (1998). Data were collected and analyzed concurrently with labelling

128 of the data beginning immediately after each interview had taken place to allow the

129 researcher to become sensitive to the incoming conceptual ideas. Transcripts were

130 reviewed line-by-line with each discreet idea or concept given a label. Where it was

131 possible, in vivo codes or direct quotations from the data were used to better represent the

132 emerging conceptual ideas (Strauss \& Corbin, 1998). Emerging concepts were clustered

133 together and then collapsed into more abstract categories as analysis progressed. During

134 the final level of analysis, the core category was selected and the relationships between

135 all other major categories were filled in. All of the initial interviews were coded by the

136 researcher and her thesis supervisor together. After the first set of interviews were

137 completed and analyzed, the findings were then reviewed with the researcher's thesis

138 supervisor and committee members together to check for logical flow of the findings. 
139 In order to ensure the quality of a grounded theory study, four criteria must be

140 present (Glaser \& Strauss, 1967, p. 237). These properties include: fitness,

141 understanding, generality, and control. The criteria of fit and understanding were met by

142 doing 'member checks' to ensure participants could provide comments on the emerging

143 theory. All of the participants were contacted within 6 months of their first interview to

144 allow for feedback and validation of emerging themes. Peer debriefing with nursing

145 colleagues also provided an avenue for reflection and evaluation. Both participants and

146 colleagues provided feedback that indicated that the theory was easy to understand and

147 'resonated' with both their personal and professional experiences.

148 The property of generality was met by gathering both sufficient amounts of data

149 from a diversity of sources which included not only information from participants but

150 also through ongoing consultation with several public health and mental health nurses.

151 The final criterion of control was attained by developing both general and specific

152 interventions to direct care around pregnancy and the postnatal period for potential users

153 of the substantive theory to help in the management of a woman with a developing or

154 present PPMD.

155 Ethical Considerations and Approval: After receiving approval from the

156 affiliated University Human Ethics Board, ethical approval was then sought from the

157 Public Health Department (PHD) Human Ethics Board of a large Canadian city. Given

158 the sensitive nature of examining PPMD's, an action plan was developed to assess

159 whether women were experiencing either no distress, mild distress, moderate distress, or

160 severe emotional distress. Women were assessed using a basic mental health status exam

161 to evaluate such factors as thought content and safety in relation to themselves and their 
162 children to determine whether inclusion within the study was appropriate. Within this

163 sample, the only intervention that was necessary for the participants was to provide

164 psycho-educational support. Informed consent was obtained from all study participants

165 and each participant was given an identification number for purposes of anonymity. The

166 tapes and transcripts have been locked in a safe location and will be destroyed 7 years

167 after completion of this research project.

\section{Findings}

169 Participants: The 10 women who participated in this study were a relatively

170 homogenous sample. They were mature (mean age $=32.6$ years with a standard deviation

171 of 2.011), well educated, Caucasian and economically middle-class. All of the

172 participants were married and English speaking with the exception of one woman whose

173 first language was French although she was fluently bilingual.

174 At the time of the first interview, 6 of the participants had 2 children, 2 of the

175 participants had 1 child but both were in their $3^{\text {rd }}$ trimester of a second pregnancy, and 2

176 of the women had a single child. The ages of the children at the time of the first interview

177 ranged from 7 months to 3 years. Two of the 6 participants who had 2 children at the

178 time of the first interview had experienced a PPMD after each baby. Two of the

179 participants with 2 children experienced a PPMD after the first baby only. One of the

180 participants with 2 children experienced a PPMD after the second child only. One

181 participant with 2 children experienced a PPMD that began after the first child and did

182 not resolve through the second pregnancy and continues to be treated. At the time of the

183 second interview, both mothers who were pregnant at the first interview had given birth

184 to their second children and both were experiencing significant symptoms of a PPMD 
185 again. Of the two remaining participants who had single children, one woman had

186 consciously decided not to get pregnant again as a direct result of her experience with a

187 PPMD. The remaining mother was in the process of planning her second pregnancy but

188 was methodically reviewing her experience to arrange and organize for the appropriate

189 resources to be in place before she would actively attempt a pregnancy again. The ages

190 of the children at the time of the second interview ranged from 3 weeks to $3 \frac{1}{2}$ years.

191 In terms of use of obstetrical service providers, 5 of the participants had shared care

192 with their family physicians and obstetricians, 2 received care from their family

193 physicians and obstetricians while employing the additional services of a doula, and the

194 remaining 3 participants used midwife services exclusively. Eight women gave birth

195 vaginally and 2 delivered by caesarean delivery. All of the participants initiated breast-

196 feeding while in hospital. Of the 10 women in the study, 8 sought out professional help

197 while 2 relied exclusively on the support of lay others for resolution of their PPMD. All

1988 who received professional attention were diagnosed as having a mood disorder and

199 were prescribed pharmacological therapy for their illness. Of the eight women prescribed

200 medication, 3 were referred on for individual counselling, 1 returned to a counsellor that

201 she had previously accessed for a separate depressive episode, and 3 participants attended

202 a weekly postpartum depression support group for auxiliary assistance in dealing with

203 their illness. At the time of the second interview, 4 of the 10 participants were continuing

204 to experience some moderately debilitating symptoms, 3 women were experiencing

205 mildly distressing symptoms and 3 participants were virtually symptom-free. 
Following a protracted involvement with the data, the core category of 'Having

208 postpartum' emerged to capture the experience of seeking help for a PPMD and refers to

209 a process across time from the inception of the mood disorder through to varying levels

210 of recovery. This is the terminology used by mothers to identify their illness. It provides

211 a label for the disease process while at the same time removing it from the mental illness

212 spectrum. There is no qualifier attached to the title. For in fact, all women who give

213 birth have 'postpartum'. This use of the label of 'having postpartum' provides safety for

214 women in a society where the stigma around mental illness remains deeply entrenched.

215 Samantha: Saying 'postpartum' just sounds better. Kind of less serious. Like it

216 still sounds bad but I don't know.... If it just wasn't so tied to the word 'depression'

217 because that is just so stigmatized.

218 Four main stressors were also identified by the women as significantly influencing

219 their mental health and contributed to their downward spiral into a PPMD. These factors

220 included: 1) An unplanned or unsupported pregnancy 2) Lack of identification of risk for

221 developing symptoms of a PPMD and/or delayed diagnosis 3) A traumatic birth

222 experience and 4) Breast feeding difficulties. Two barrier categories and a single enabler

223 category were further identified by the women as influencing their help-seeking

224 behaviours. The barrier categories include: 1) stigma and 2) health care provider issues.

225 The enablers identified by women were collapsed into a single category that was all

226 encompassing titled 'comprehensive maternal-child care' which fundamentally

227 represented a philosophy that fully embraces holistic care from health care providers.

228 Significant stressors that contributed to the development of a PPMD 
Pregnancy is in and of itself a significant emotional and physical event in a

230 woman's life. The stressors that women who participated in this study identified as

231 contributing to the development of a PPMD are consistent with those found in the

232 literature (Beck, 2004; Cohen \& Nonacs, 2005; Forman, Videbeck, Hedegaard, Salvig \&

233 Secher, 2000; The Maternity Centre Association, 2002). Some participants talked about

234 the experience of an unplanned or unsupported pregnancy resulting in considerable

235 emotional turmoil and ambivalence leaving the mother with a wash of negative emotions

236 while laying the foundation for the beginning symptoms of a mood disorder (Rich-

237 Edwards, et al., 2006). For some women in the present study, a previous history of a

238 PPMD or symptoms of a mental illness were clearly developing or were exacerbated

239 during the pregnancy and were not identified or treated in a timely fashion. This has also

240 been shown to be a strong predictor of the development of a PPMD in the literature

241 (Dennis \& Chung-Lee, 2006; Watt, Sword, Krueger, \& Sheehan, 2002). Many women

242 identified what they would describe as a traumatic birth experience as the single greatest

243 event that triggered the beginning of the spiral into the darkness that defines the illness

244 (Beck, 2004; Benoit, Westfall, Treloar, Phillips, \& Jansson, 2007). Finally, a number of

245 participants also identified breast-feeding as a tremendous stressor that contributed

246 significantly to the development or worsening of symptoms. All of the participants

247 attempted to breast-feed and stated that the "extreme societal pressure" to breast-feed at

248 any cost because all 'good' mothers should very much impacted their decision to start

249 and provided tremendous guilt in the event that they felt unable to continue (Wall, 2001).

250 Being aware of and attentive to these stressors for women which may contribute to the

251 development of a PPMD is critical and has significant implications for nursing practice. 


\section{Barrier categories to seeking help for a PPMD}

253 The category of stigma represents an in vivo code with women speaking often of

254 the shame associated with having a mental illness. As a result of the stigma and shame

255 that continues to plague those with mental illness, the mothers identified it as a significant

256 barrier to reaching out to both professional and lay help which often begins only when

257 symptoms are no longer manageable. When rebuffed by others for expressing their

258 concerns, women were left shaken and forced to manage symptoms on their own.

259 Invalidated feelings by a close significant other were particularly painful.

260 Maggie: I think the biggest one was with my husband. I was worried that he would

261 look at me as "Oh, she's not doing a very good job” and my in-laws too "Oh, she can’t

262 cope and she's not doing a very good job as a mother" and I always had in the back of my

263 mind that if something ever happened between my husband and I that he would get the

264 kids cause I'm just not doing a good job.

265 The category of health care provider issues developed as a result of no single

266 discipline being responsible for women's mental health concerns during the perinatal

267 period. Despite frequent contacts with various health care providers (e.g. physicians,

268 nurses, midwives) symptoms go largely unnoticed without one discipline specifically

269 addressing and monitoring these issues. It also leads to confusion for women as to who

270 they should reach out to for professional help. Without professional ownership for

271 assessing and managing mental health of women throughout the perinatal period,

272 symptoms went largely unnoticed.

273 Lynn: My six-week visit? It was a waste of time. An absolute waste of time. She

274 went in, I got weighed, she saw the baby and said "Oh, how cute!" and then that was it. 
275 Absolutely no questions about how I was feeling at all, at all. It was a social tea. It was a 276 social tea with somebody who is really afraid to ask you if you are feeling "blue" because 277 then you will take up too much of their time.

278 As an extension of health care provider issues, a lack of knowledge/ awareness was 279 demonstrated by many health care providers and laypersons about PPMD's. PPMD's can 280 configure and present themselves in many different ways and often begin prenatally. A 281 lack of knowledge about when these illnesses may begin and the extreme variability of 282 symptom patterns may leave practitioners and significant others unprepared to identify 283 problems as they appear. Lack of knowledge/awareness about PPMD's was also 284 apparent once a PPMD was identified as pharmacological intervention was offered as the 285 only solution to most of the women who sought professional help. Few were given the 286 option of supportive conjunctive therapy. This was a significant barrier for women as 287 they described a strong distaste for pharmacological intervention and often delayed the 288 women from seeking treatment because they felt that they would be offered medications 289 for symptom management and that this would solidify the depth of their illness, prevent 290 them from continuing breastfeeding or be the only option available to them.

291 Darcy: So, I went for my six-week postpartum check-up at eight weeks. I was a

292 couple of weeks late but I saw the guy, you know, the OB who did the c-section and he

293 just basically looked at me and like I was bawling in his office and crying and telling 294 about my experience and my fears and he gave me a prescription for antidepressants then 295 he just wrote me a bunch of repeats for the Zoloft and offered no-follow-up.

296 Mothers strongly felt that pharmacological intervention alone was unsuitable and 297 served as a 'quick fix' or 'band-aid' to the reality of their illness. For these mothers, the 
298 fear of treatment served as a significant barrier and impacted their decisions when

299 determining if and when to seek professional help.

300 Eventually, for many women, the symptoms worsened and the length of time

301 between episodes became shorter making the need to get help more apparent. This is a

302 significant barrier for women in seeking help and is linked to the category of health care

303 provider issues in that the periods of waxing and waning would be less deleterious if a

304 professional group was monitoring a mother's mental health over a period of time to

305 capture the big picture.

$306 \quad$ Enabler categories to seeking help for a PPMD

307 The enabler category of comprehensive maternal-child care reflects a system of

308 care that fully meets the physical, psychological, and emotional needs of the mother-child

309 dyad. By shifting care beyond the physical parameters of obstetrical care, women felt it

310 would create a safe place for them to seek help for their symptoms through open

311 dialoguing and ultimately reduce the stigma and hidden nature of mental illness during

312 the perinatal period. By working to shift society's views around mental illness and

313 mothering, receiving help would not be seen as a failing but as 'normal'. This was

314 described as a significant enabler to the help seeking process and is reciprocal in nature to

315 the barrier category of stigma.

316 Samantha: I don't even remember it coming up [prenatally]. Maybe just briefly.

317 But ya, certainly if they made moms and their partners more aware of the possibility and

318 where to go if it does happen and stuff. I guess just really make it open and there. Make

319 it okay to talk about. This whole mental illness thing is really just so stigmatized and it's

320 really sad. 
321 As part of comprehensive maternal-child care and the transition to making mental

322 health care an equal priority to the physical care that is given was a strong desire to

323 integrate screening for PPMD's. Just as screening for diabetes prenatally is a standard

324 part of care, women want screening for mental health to be integrated as the standard of 325 care.

326 Zola: I think it needs to be a standardized thing. Just like a gestational diabetes

327 screen. You know, they don't just do it on the fat women; they do it on the skinny

328 women, right? If it was a standard thing that they gave every woman between 36 and 40

329 weeks and that they continued to do it during the follow-up that would be good.

330 For all of the women who participated in this study, none were screened with a

331 screening tool for a mood disorder at any time during the perinatal period. This would

332 have served as a significant enabler to identifying the presence of symptoms earlier in the

333 course of the illness as women felt that the implementation of a standardized system of

334 screening for depression or mental illness in general should be a regular part of their care.

335 The integration of screening as part of a system of comprehensive maternal-child care

336 responds to many of the elements found within the barrier category of health care

337 provider issues.

338 The final significant element of truly comprehensive maternal-child care system,

339 requires that health care providers embrace mothers and babies as a single unit of care

340 such that women feel equally valued to their children. In creating this environment,

341 mothers would feel that their concerns would be validated and seen as a similar priority.

342 Through adhering to this philosophy, women would have their concerns validated early 
343 and often. This would greatly enable the process of feeling safe to disclose the illness

344 experience.

345 Zola: It's not about you. It's not about me. It's not about the woman that is having

346 the baby. They are just there to make sure that the baby is okay, the baby is growing, and

347 it's all about the baby. The baby, the baby, the baby.

The clinical implication of these findings for nursing requires that we alter our

350 practice to more adequately meet the needs of women during pregnancy and in the

351 postnatal period. It is important that we acknowledge stressors that women have

352 identified as contributing to the development of their PPMD. This should direct our

353 practice by giving us important opportunities to ask women without judgment about the

354 emotional impact of their pregnancy, previous mental health issues, birth experiences,

355 and breast-feeding issues. This may provide helpful insight into the mental well-being of

356 a woman and allow for open and safe discussions in various clinical contexts. The

357 continuing stigma and shame associated with having a psychiatric illness serves as a

358 significant barrier for women to seek out help from both lay and professional others when

359 experiencing a PPMD (Pinto-Foltz \& Logsdon, 2008). In this study, the mothers spoke

360 often of the stigma around mental illness that forced them to hide and mask their

361 symptoms and negative feelings around mothering ultimately diminishing their quality of

362 life. At the same time, in delaying their treatment to avoid judgement from others, they

363 identified that their interactions with their children were often markedly compromised.

364 Maternal mood disorders greatly diminish the quality of the interaction between the dyad

365 ultimately resulting in emotional, social, and cognitive delays for their children (Luoma, 
366 Tamminen, Kaukonen, Laippala, \& Puura et al., 2001). The outcomes for children are

367 particularly compromised and correlated with the chronicity of their mother's illness

368 indicating that delayed treatment has significant long-term implications for the dyad and

369 family unit as a whole (Hay, Pawlby, Sharp, Asten \& Mills et al., 2001). These findings

370 highlight the urgency in detecting and treating mental illness within this population while

371 also giving strong evidence that women want to be asked open and direct questions

372 around their mental health.

373 The finding that maternity care should be delivered within the context of the

374 mother-child dyad as the unit of care is an important one. Our current system often places

375 primary focus on the health and wellness of infants to the exclusion of their mothers.

376 Women need their physical health monitored appropriately but also need emotionally

377 supportive care that values and honours the needs of both women and their babies. This

378 philosophy of care highlights the necessity for a holistic approach with the dyad together

379 as a focus throughout this life event (Health Canada, 2002).

380 Women very clearly identified that they wanted universal screening for mood

381 disorders to be implemented as part of the standard of practice for maternity care.

382 Screening for mood disorders is a simple and economical process that is well within the

383 scope of our nursing practice allowing us to take ownership for not only its

384 implementation but also to be leaders in advocating for this best practice standard to be

385 available to all women. The design and delivery of mass screening programs for

386 perinatal mood disorders will require the concerted effort of maternal-child nurses, public

387 and community health nurses and other nurses who work directly with this population. 
389 generalizeable beyond the present participant sample. The mothers who participated

390 within this study were all well educated, white, mature (mean age $=32.6$ ), professional

391 women with few financial or social limitations. Further, all of the participants had

392 partners who were able to provide at least minimal instrumental and emotional support.

393 For new mothers without these financial, emotional, and social benefits, the experience of

394 a PPMD would undoubtedly be more profound and complex (Beck, 1996). As a result,

395 the study findings must be transferred to other populations with considerable caution.

\section{Conclusion}

397 In listening to women's voices, we now have a greater understanding of the barriers

398 that prevent them from seeking help for a PPMD. Further, by directly asking mothers

399 how the process can be enabled, the women have provided solutions towards improving

400 care around their mental health needs during this important development life stage. This

401 may be best achieved through providing screening for all women across the perinatal

402 period. This should also facilitate making women's mental health a priority within the

403 context of a safe and open environment for disclosure of symptoms while enabling

404 women to reclaim their mothering souls. 


\section{References}

American Psychiatric Association (2000). Diagnostic and statistical manual of mental disorders (4 ed, TR). Washington, DC.

Barr, J.A. (2006). Postpartum depression, delayed maternal adaption, and mechanical infant caring: A phenomenological hermeneutic study. International Journal of Nursing Studies 45(2), 362-369.

Beck, C.T. (1996). A meta-analysis of predictors of postpartum depression. Nursing Research, 45(5), 297-303.

Beck, C.T. (1999). Postpartum depression: Stopping the thief that steals motherhood. AWHONN Lifelines, 3(4), 41-44.

Beck, C.T. (2004). Birth trauma: In the eye of the beholder. Nursing Research, 53(1). 28-35.

Beck, C.T. \& Driscoll, J.W. (2006). Postpartum mood and anxiety disorders: A clinician's guide. Sudbury, MA: Jones and Barlett Publishers.

Bryant, A., \& Charmaz, K. (2007). The Sage handbook of grounded theory. Thousand Oaks, CA: SAGE Publications Inc.

Charmaz, K. (2006). Constructing grounded theory: A practical guide through qualitative analysis. Thousand Oaks, CA: Sage Publishing Inc.

Cohen, L.S. \& Nonacs, R.M. (Eds). (2005). Mood and anxiety disorders during pregnancy and postpartum. Washington, DC: American Psychiatric Publishing, Inc.

Dennis, C-L., Chung-Lee, L. (2006). Postpartum depression help-seeking barriers and maternal treatment preferences: A qualitative systematic review. Birth, 33(4), 323-331. 
Field, T. (2010). Postpartum depression effects on early interactions, parenting and safety practices: A review. Infant Behaviour and Development, 33, 1-6.

Forman, D.N., Videbech, P., Hedegaard, M., Salvig, J.D. \& Secher, N.J. (2000). Postpartum depression: Identification of women at risk. British Journal of Obstetrics and Gynaecology, 107, 1206-1210.

Gao, W., Paterson, M., Abbott, S. \& Carter, L.I. (2007). Maternal mental health and child behaviour problems at 2 years: Findings from the Pacific Islands Families Study. Australian and New Zealand Journal of Psychiatry, 41, 885-895.

Glaser, B.G. \& Strauss (1967). The discovery of grounded theory: Strategies for qualitative research. Aldine De Gruyter: NY

Gold, L. H. (2002). Postpartum disorders in primary care. Primary care: Clinics in office practice, 29, 27-41.

Goodman, J.H. (2004). Postpartum depression beyond the early postpartum period. Journal of Obstetric, Gynecologic, and Neonatal Nursing, 33, 410-420.

Hayes, B. (2010). From 'postnatal depression' to perinatal anxiety and depression': Key points of the National Perinatal Depression Plan for nurses and midwives in Australian primary health care settings. Contemporary Nurse 35(1), 58-67.

Hay, D.F., Pawlby, S., Sharp, D., Asten, P., Mills, A. \& Kumar, R. (2001). Intellectual problems shown by 11-year-old children whose mother had postnatal depression. Journal of Child Psychology and Psychiatry, 42, 871-889.

Health Canada (2002). Family-Centred Maternity and Newborn Care: National Guidelines. www.hc-sc.gc.ca

Holopainen, D. (2002). The experience of seeking help for postnatal depression. Australian Journal of Advanced Nursing, 19(3), 39-44. 
Luoma I., Tamminen, T., Kaukonen, P., Laippala, P., Puura, K., Salmelin, R. et al. (2001). Longitudinal study of maternal depressive sysmptoms and child wellbeing. Journal of the Anmerican Academy of Child \& Adolescent Psychiatry, 40(12), 1367-1374.

Mancini, F., Carlson, C. \& Albers, L. (2007). Use of postpartum depression screening scale in a collaborative obstetric practice. Journal of Midwifery \& Women's Health 52(5), 429-434.

Mason, T., Carlisle, C., Watkins, C \& Whitehead, E (Eds). (2001). Stigma and Social Exclusion in Healthcare. London, UK: Routledge.

McCreaddie, M., \& Payne, S. (2010). Evolving grounded theory methodology: Towards a discursive approach. International Journal of Nursing Studies, 47, 781-793.

Meleis, A. I. (2005) Theoretical nursing: Development and progress ( $3^{\text {rd }}$ Ed.). Philadelphia: Lippincott, Williams \& Wilkins.

Meighan, M., Davis, M.W., Thomas, S.P., Droppleman, P.G. (1999). Living with postpartum depression: The father's experience. MCN, The American Journal of Maternal/Child Nursing, 24(4),202-208.

Nonacs, R.M. (2005). Postpartum mood disorders. In Cohen, L.S. \& Nonacs, R.M. (Eds). Mood and anxiety disorders during pregnancy and postpartum. Washington, DC: American Psychiatric Publishing, Inc.

Pearlstein, T., Howard, M., Salisbury, \& Zlotnick, C. (2009). Postpartum depression. American Journal of Obstetrics and Gynecology, April, 357-364.

Pearson, R.M., Cooper, R.M., Penton-Voak, I.S., Lightman, S.L., Evans, J. (2010). Depressive symptoms in early pregnancy disrupt attention processing of infant emotion. Psychological Medicine, 40, 621-631. 
Pinto- Foltz, M.D., Logsdon, M.C. (2008). Stigma towards mental illness: A concept analysis using postpartum depression as an exemplar. Issues in Mental Health Nursing, 29, 21-36.

Rich-Edwards, J.W., Kleinman, K., Abrams, A., Harlow, B.L., McLaughlin, T.J. Joffe, H., Gillman, M.W. (2006). J Epidemiol Community Health, 60, 221-227.

Strauss, A. \& Corbin, J. (1998). Basics of qualitative research: Techniques and procedures for developing grounded theory (2 $2^{\text {nd }}$ ed.). Sage, CA.

The Maternity Center Association (2002). Listening to mothers: The first national U.S. survey of women's childbearing experiences.

Tough, S.C., Siever, J.E., Leew, S., Johnston, D.W., Benzies, K. \& Clark, D. (2008). Maternal mental health predicts risk of developmental problems at 3 years of age: Follow up of a community based trial. BMC Pregnancy and Childbirth, 8(16).

Tronick, E. (2007). The neurobehavioral and social-emotional development of infants and children. New York, NY: W.W. Norton \& Company, Inc.

Ugarriza, D. N. (2002). Postpartum depressed women's experience of depression. Journal of Nursing Scholarship, 34, 227-233.

Wall, G. (2001). Moral constructions of motherhood in breastfeeding discourse. Gender and Society, 15(4), 592-610

Watt, S., Sword, W., Krueger, P., \& Sheehan, D. (2002). A cross-sectional study of early identification of postpartum depression: Implications for primary care providers from The Ontario Mother \& Infant Survey. BMC Family Practice, 3. http://www.biomedcentral.com/1471-2296/3/5 